\title{
A Carbon star approach to IC 10: Distance and correct size ${ }^{\star, \star \star}$
}

\author{
S. Demers ${ }^{1}$, P. Battinelli ${ }^{2}$, and B. Letarte ${ }^{3}$ \\ ${ }^{1}$ Département de Physique, Université de Montréal, CP 6128, Succursale Centre-Ville, Montréal, Québec H3C 3J7, Canada \\ e-mail: demers@astro.umontreal.ca \\ 2 INAF - Osservatorio Astronomico di Roma, Viale del Parco Mellini 84, 00136 Roma, Italia \\ e-mail: battinel@oarhp1.rm.astro.it \\ 3 Kapteyn Astronomical Institute, Posbus 800, Groningen, 9700 AV, The Netherlands \\ e-mail: bruno@astro.rug.nl
}

Received 29 March 2004 / Accepted 26 May 2004

\begin{abstract}
We present wide field CFH12K observations of the highly reddened Local Group galaxy IC 10 . Using $R, I$, $\mathrm{CN}$ and TiO filters we identify 676 carbon stars in the field of IC 10. After mapping the reddening in the field, from the colours of the G dwarfs seen along the line of sight, we determine the mean apparent magnitude of the C star population to be $\left\langle I_{0}\right\rangle=19.78$, leading to a true modulus of $(m-M)_{0}=24.35 \pm 0.11(741 \pm 37 \mathrm{kpc})$. The old red giant stars define an asymmetric halo. With a halo diameter of at least 30', IC 10 is among the largest dwarfs of the Local Group. The surface density of C stars follows a radial power law with a scale length of $2.36^{\prime}$, a value nearly identical to the scale length defined by the old giants thus IC 10 has a stellar halo where the old and intermediate-age populations are well mixed.
\end{abstract}

Key words. galaxies: individual: IC 10 - stars: carbon - galaxies: local group - galaxies: structure

\section{Introduction}

According to van den Bergh's (2000) interesting Local Group review, IC 10 was recognized as an extragalactic object by Mayall (1935). One year later Hubble (1936) suggested that it might be a Local Group member. However, his other two suggested members IC 342 and NGC 6946, never joined the Local Group club. IC 10 is located at $b=-3.3^{\circ}$ thus is it not only dimmed considerably by interstellar extinction but its outlines are confused by the high foreground stellar density. This unfortunate fact handicapped so much the optical investigations of IC 10 that during the first decades, following its recognition, only one photometric result appears. De Vaucouleurs \& Ables (1965) obtained its integrated magnitude $(V=10.4 \pm 0.2)$ and determined its reddening $(E(B-V)=0.87)$ from its integrated $(U-B)$ and $(B-V)$ colours. During that period, IC 10 was targeted by radio astronomers, Huchtmeier (1979) found that the HI envelope of IC 10 reaches $62^{\prime} \times 80^{\prime}$, a size much larger than its optical image whose dimension are $5.5^{\prime} \times 7.0^{\prime}$ (Massey \& Armandroff 1995). In this respect, IC 10 and NGC 6822

* Based on observations carried out at the Canada-France-Hawaii Telescope, operated by the National Research Council of Canada, the Centre National de la Recherche Scientifique de France, and the University of Hawaii.

$\star \star$ Full Table 2 is only available in electronic form at the CDS via anonymous ftp to cdsarc.u-strasbg.fr $(130.79 .128 .5)$ or via http://cdsweb.u-strasbg.fr/cgi-bin/qcat?]/A+A/424/125 are two Local Group irregulars with a huge HI envelope, like NGC 3109 which belongs to the nearby Antlia - Sextans group (van den Bergh 1999).

Table 1 presents a 40-year compilation of the published distance and reddening estimates. This list shows that recent distances range from $500 \mathrm{kpc}$ to $1 \mathrm{Mpc}$. The reddening $E(B-V)$ inside of IC 10, determined from its Population I objects, seems to be larger than 1.0, while the foreground reddening is closer to 0.80 .

Our Local Group carbon star survey has shown (Demers et al. 2003) that the mean I magnitude of late C stars can be used as a standard candle. Thus we can adopt this approach to obtain the distance of IC 10, providing that the $\mathrm{C}$ star apparent magnitudes can be corrected for the interstellar extinction. To achieve this goal, the reddening toward IC 10 is evaluated from the whole CMD of the observed area.

\section{Observations}

The results presented here are based on observations obtained, in Service Queue observing mode in August 2002, with the CFH12K camera installed at the prime focus of the $3.66 \mathrm{~m}$ Canada-France-Hawaii Telescope. The camera consists in a $12 \mathrm{k} \times 8 \mathrm{k}$ pixel mosaic covering a field of $42^{\prime} \times 28^{\prime}$, each pixel corresponding to 0.206 arcsec. Images were obtained through Mould $I$ and $R$ filters and narrow band $\mathrm{CN}$ and TiO filters, centered at $808.6 \mathrm{~nm}$ and $768.9 \mathrm{~nm}$, respectively. Exposures 
Table 1. Historic summary of IC 10 distance and reddening determinations.

\begin{tabular}{lcll}
\hline \hline Mpc & $E(B-V)$ & Method & Reference \\
\hline 1.25 & & HII regions & de Vaucouleurs \& Ables (1964) \\
& 0.87 & integrated UBV & de Vaucouleurs \& Ables (1964) \\
3.0 & & HII regions & Sandage \& Tamman (1974) \\
2.0 & 0.40 & HII regions & de Vaucouleurs (1978) \\
& 0.47 & Balmer decrement & Lequeux et al. (1979) \\
1.8 & & PN & Jacoby \& Lesser (1981) \\
2.0 & & Tully-Fisher & Bottinelli et al. (1984) \\
2.2 & & PN & Ciardullo et al. (1989) \\
& & H $/$ radio ratio & Yang \& Skillman (1993) \\
1.04 & 0.93 & blue \& red stars & Karachentsev \& Tikhonov (1993) \\
0.95 & 0.80 & Wolf-Rayet & Massey \& Armandroff (1995) \\
0.83 & 0.97 & Cepheids & Saha et al. (1996) \\
0.82 & & IR Cepheids & Wilson et al. (1996) \\
0.66 & 1.16 & Cepheids & Sakai et al. (1999) \\
0.50 & 0.85 & TRGB & Sakai et al. (1999) \\
0.58 & 0.99 & $V, R, I$ & Tikhonov (1999) \\
0.59 & 1.05 & $J, H, K$ & Borisova et al. (2000) \\
0.98 & 0.77 & spectra HII reg. & Richer et al. (2001) \\
0.74 & Variable & C stars & Hunter (2001) \\
\hline
\end{tabular}

for each filter were three $450 \mathrm{~s}$, eight $475 \mathrm{~s}$, three $1200 \mathrm{~s}$ and three $1200 \mathrm{~s}$ respectively, under excellent seeing. The total exposure time was less than $3.5 \mathrm{~h}$.

The data distributed by the CFHT have been detrended. This means that the images have already been corrected with the master darks, biases, and flats. Fringes have been removed on $I$ exposures under $60 \mathrm{~s}$ and large scale structures such as the "Skyring" effect have been removed when relevant. This preanalysis produces 12 CCD images, of a given mosaic, with the same zero point and magnitude scale.

We carefully combine images taken with the same filter, making sure that the final FWHM was similar to the average FWHM. The photometric reductions were done by fitting model point-spread functions (PSFs) using DAOPHOT-II/ALLSTAR series of programs (Stetson 1987, 1994). Details of the photometric calibration have been described in recent papers of this series (see, for example Demers et al. 2003).

\section{Results}

The $I$ vs. $R-I$ colour-magnitude diagram (CMD) for the whole field is displayed in Fig. 1 where $\approx 90000$ stars with photometric colour errors less than 0.1 are plotted. The CMD reaches $I \approx$ 23 , an extended giant branch appear above this limit. The other conspicuous feature of the diagram is the sloping vertical ridge starting at $I=16,(R-I)=0.6$ and reaching $(R-I)=1$ and $I \sim 21$. This ridge corresponds to Galactic G dwarfs located at the main sequence turnoff and seen along the line of sight. Normally, for directions with low extinction, this ridge is vertical and shifted according to the reddening, see for example the CMD of NGC 6822 (Letarte et al. 2002), but here the reddening increases with distance and fainter $\mathrm{G}$ stars are more reddened than the brighter ones. The colour position of this feature can be used to map the reddening across the field. A similar sloping ridge is seen in the CMD of IC 10 presented by Sakai et al. (1999), see their Fig. 2c.

\subsection{The reddening toward IC 10}

In order to investigate the differential reddening across the IC 10 field, we divide the whole field into 24 cells of $2 \mathrm{k} \times 2 \mathrm{k}$ pixels (smaller cells were often found to have too few stars in each magnitude interval for robust statistics). The widening of the sloping feature visible in the CMD of Fig. 1 is more clearly seen in Fig. 2 which corresponds to a representative cell, outside of the core of IC 10 . This widening is undoubtedly produced by the combined effects of differential reddening and photometric errors which become larger toward the limiting magnitudes. The broken line corresponds to the mid-ridge, defined as the peak of the colour distribution at various magnitude intervals. This ridge, which can be easily followed down to $I \sim 21$, represents Galactic G dwarfs located on their main sequence turnoff seen at increasing distance and reddening along the line of sight. These stars have intrinsic colour $(R-I)_{0}=0.35(\operatorname{Cox} 2000)$. For $I=21$, with a 


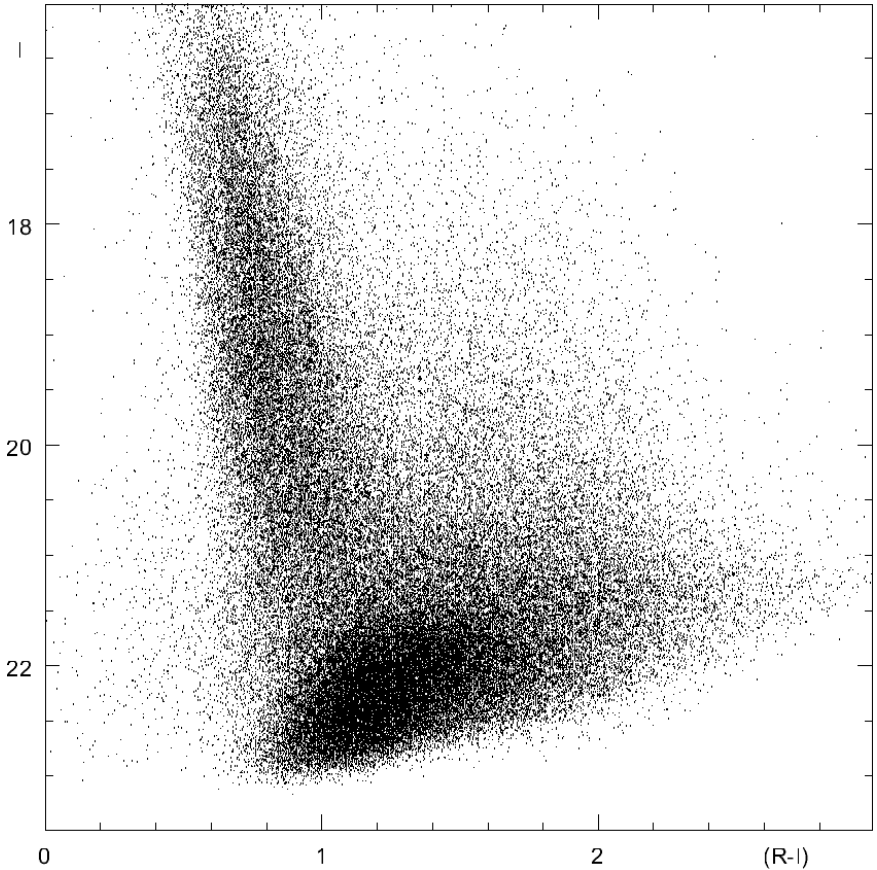

Fig. 1. Colour-magnitude diagram of the whole CFH12K field. Stars with colour errors $<0.10$ are plotted. A substantial differential reddening is responsible of the fuzziness of the features.

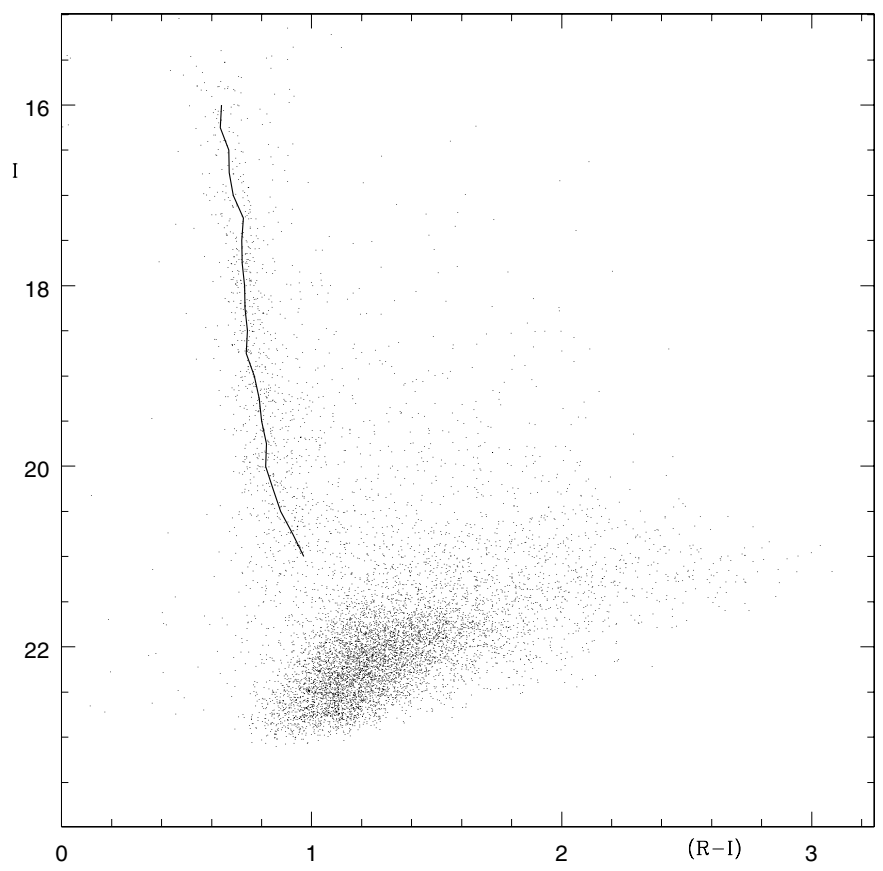

Fig. 2. Colour-magnitude diagram of a representative cell showing better defined features. The line corresponds to the mid-ridge, see text for details.

reddening of $\sim 0.60$ a $\mathrm{G}$ dwarf of $M_{I}=+3.6$ would be located at $\sim 14 \mathrm{kpc}$. At this distance, the line of sight in the direction of IC 10 , would be some $800 \mathrm{pc}$ above the Galactic plane. Is it reasonable to interpret the slope of the ridge as a continuous increase in reddening, up to this distance above the plane? The Galactic longitude of IC 10 is $118^{\circ}$, we are thus looking at some $25 \mathrm{kpc}$ from the Galactic center.
IRAS observations (of warm dust) yield a scale height of $120 \mathrm{pc}$ for the exponential dust layer, see Deul \& Burton (1992) for review. Subsequent observations at longer wavelengths by COBE/DIRBE (Sadroski et al. 1994), ISO (Alton et al. 1998) and submillimetric observations (Alton et al. 2001) have shown that spiral galaxies have extended distribution of cold dust which reaches further out than the stellar disk. Optical and near infrared modeling of edge-on spirals by Xilouris et al. (1999) reveals that the dust scale heights are about half the stellar scale heights. Furthermore, the modeling of NGC 891 (Xilouris et al. 1998), a spiral quite similar to the Milky Way, requires two stellar and two dust disks to fit the surface brightness of the galaxy. It is then reasonable to assume that the Galaxy possesses two layers of dust, one corresponding to the thin stellar disk and a more tenuous one corresponding to the thick disk. The stellar scale heights of the thin and thick stellar disks have been evaluated by Méndez \& Guzmán (1998) from star counts in the Hubble deep field. They quote $250 \mathrm{pc}$ for the thin disk and two solutions for the thick disk: $1300 \mathrm{pc}$ with a $2 \%$ contribution or 750 pc with a $6 \%$ contribution.

We determined that the observed reddening increase with apparent magnitudes is compatible, within $\pm 0.05 \mathrm{mag}$, with a single exponential dust layer with a scale height of $250 \mathrm{pc}$. We stress that better solutions are certainly possible, however we did not investigate multi-layer solutions because the number of free parameters involved makes this exercise unproductive. Furthermore, the likelihood that the scale height varies with galactocentric distances introduces a serious complication to the modeling.

In order to reduce, as much as possible, the reddening discontinuity between the cells we also evaluate the reddening in $152 \mathrm{k} \times 2 \mathrm{k}$ cells centered on the intersections of the grid. We deredden each star by calculating the weighted mean distance of the star to its nearest three cells, we then calculate its extinction from this reddening. We summarize the reddening determinations by presenting a reddening map in Fig. 3. The reddening varies, in a irregular way, by as much as 0.30 mag across the field. The mean reddening in the field is $E(R-I)=0.63$ which translate into $E(B-V)=0.79$ and $A_{I}=1.51$, using the reddening law by Cardelli et al. (1989) adjusted for the CFHT Mould's filters. This average colour excess represents the reddening of the periphery of IC 10 since its estimate is based mostly on stars away from the central core of IC 10. It agrees well with some recent determinations, listed in Table 1. The application of these reddenings and extinctions yields the reddening corrected colour-magnitude diagram displayed in Fig. 4; it is evident how the sloping ridge is now narrower than that shown in Fig. 1. The slope of the ridge is still present because by applying the maximum reddening we over-correct the nearby brighter stars.

\subsection{Carbon star identification}

Carbon stars are selected from their location in the $(\mathrm{CN}-\mathrm{TiO})$ vs. $(R-I)_{0}$ plane. The colour-colour diagram is displayed in Fig. 5. Throughout this series of papers we have selected as an adoption criterion, stars with $\sigma<0.125$, 


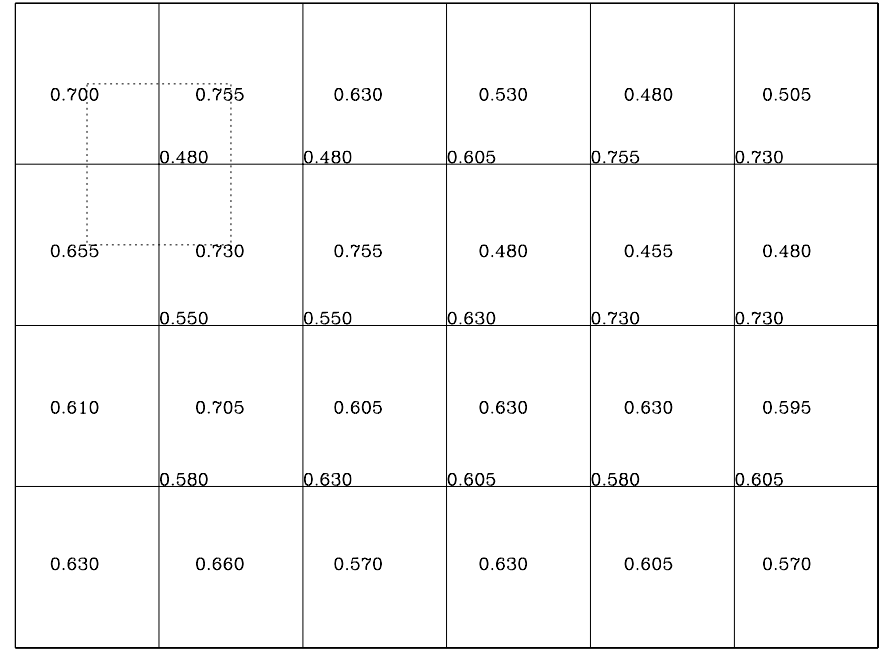

Fig. 3. The mean $E(R-I)$ in each of the $2 \mathrm{k} \times 2 \mathrm{k}$ cells. The dashed box represents one of the 15 additional cells whose adopted reddening is given near the intersections.

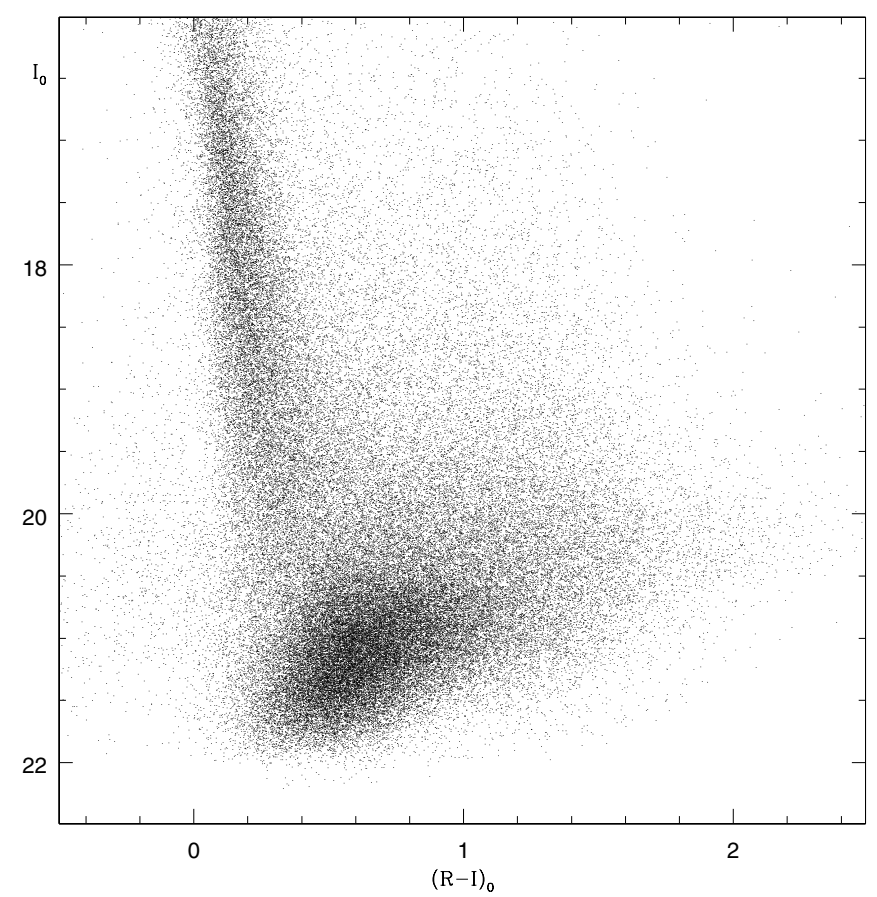

Fig. 4. The reddening corrected colour-magnitude diagram of the whole region.

where $\sigma=\left(e_{R-I}^{2}+e_{\mathrm{CN}-\mathrm{TiO}}^{2}\right)^{1 / 2}$. Over 66000 stars in the field satisfy this criterion. The zero point of the $(\mathrm{CN}-\mathrm{TiO})$ index is set according to the procedure outlined by Brewer et al. (1995). We set the mean of $(\mathrm{CN}-\mathrm{TiO})=0.0$ for all blue stars since hot stars are expected to have a featureless spectra in the $\mathrm{CN}$ and $\mathrm{TiO}$ regions. We thus define a blue star, as in Letarte et al. (2002), i.e. as a star in the color range $0.0<(R-I)_{0}<0.45$. The $\mathrm{CN}-\mathrm{TiO}$ approach requires a previous estimate of the local colour excess.

The upper branch of the colour-colour diagram shows that IC 10 contains several hundreds $C$ stars. Since the $\mathrm{CN}-\mathrm{TiO}$ technique confuses $\mathrm{M}$ and $\mathrm{C}$ stars for spectral types earlier than M0, we restrict our definition of $\mathrm{C}$ stars as those with $(R-I)_{0}>0.90$, a colour corresponding to M0: 676 stars

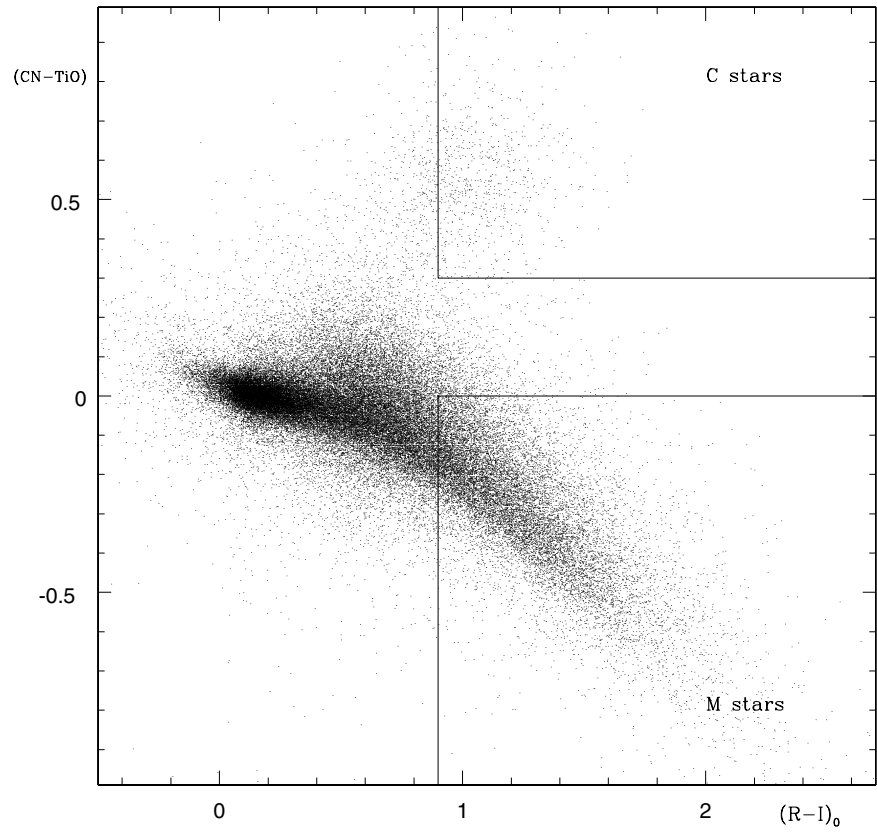

Fig. 5. Colour-colour diagram to identify $\mathrm{C}$ stars from $\mathrm{M}$ stars.

are in the C star box. The equatorial coordinates (J2000), the dereddened magnitudes and colours of these stars are listed in Table 2. It is, however, evident from Fig. 5 that in addition numerous bluer $\mathrm{C}$ stars are also present in IC 10.

Among the $\mathrm{C}$ stars retained there is a bright star with $I_{0}=16.33(I=17.86)$. This star is well outside the central core of IC 10. It is however very close to an even brighter star, saturated in our images, whose profile may be overlapping with the $\mathrm{C}$ star. The photometric errors of the $\mathrm{C}$ star are abnormally high for its magnitude, they are barely below the threshold of acceptability. A search through the 2MASS database yields a star, $3.4^{\prime \prime}$ from it, with $K=12.125$ and $J-K=1.07$. It is not clear if this corresponds to the bright star or to our $\mathrm{C}$ star. Nevertheless, the 2MASS source is too blue to correspond to a C star, as we define them (Demers et al. 2002).

\section{Discussion}

\subsection{The distance of IC 10}

The luminosity function of the $676 \mathrm{C}$ stars, located within the C star box of the colour-colour diagram, is presented in Fig. 6 . A Gaussian, with a $\sigma=0.40 \mathrm{mag}$. is eye fitted to the distribution. The mean magnitude of these $\mathrm{C}$ stars is $\left\langle I_{0}\right\rangle=19.78$ while their mean colour, $\langle R-I\rangle_{0}=1.11$. Demers et al. (2003) have shown that the $\left\langle M_{I}\right\rangle$ of $\mathrm{C}$ stars in galaxies having more than one hundred of them, shows very little dispersion. Since that paper we have observed Wolf-Lundmark-Melotte (Battinelli \& Demers 2004a), NGC 185 (Battinelli \& Demers 2004b) and NGC 147 (Battinelli \& Demers 2004c). Taking into account these new data we obtain, for galaxies with more than $100 \mathrm{C}$ stars: $\left\langle M_{I}\right\rangle=-4.57 \pm 0.09$. The uncertainty attached to $\left\langle I_{0}\right\rangle$ depends on the uncertainty of the zero point of the photometry and on the accuracy of the adopted mean $E(R-I)$. Assuming that both of these are less than 0.05 mag leads to 
Table 2. Full table is available at the CDS. A portion is shown here for guidance regarding its form and content. Units of right ascensions are hours, minutes and seconds, and units of declination are degrees, arcminutes and arcseconds.

\begin{tabular}{ccccccccc}
\hline \hline Id & RA & Dec & $I_{0}$ & $\sigma_{I}$ & $(R-I)_{0}$ & $\sigma_{(R-I)}$ & $(\mathrm{CN}-\mathrm{TiO})$ & $\sigma_{(\mathrm{CN}-\mathrm{TiO})}$ \\
\hline 1 & $0: 18: 26.58$ & $59: 19: 51.60$ & 19.806 & 0.024 & 0.957 & 0.039 & 0.511 & 0.069 \\
2 & $0: 18: 35.58$ & $59: 25: 07.00$ & 19.315 & 0.018 & 1.131 & 0.031 & 0.315 & 0.061 \\
3 & $0: 18: 37.60$ & $59: 17: 31.70$ & 19.771 & 0.024 & 0.913 & 0.038 & 0.497 & 0.049 \\
4 & $0: 18: 41.43$ & $59: 22: 18.80$ & 19.691 & 0.025 & 0.998 & 0.039 & 0.684 & 0.051 \\
5 & $0: 18: 42.73$ & $59: 18: 04.90$ & 19.961 & 0.030 & 1.066 & 0.051 & 0.540 & 0.080 \\
6 & $0: 18: 45.95$ & $59: 11: 26.90$ & 19.175 & 0.015 & 0.934 & 0.020 & 0.334 & 0.031 \\
7 & $0: 18: 48.46$ & $59: 19: 53.80$ & 19.574 & 0.025 & 1.110 & 0.040 & 0.505 & 0.049 \\
8 & $0: 18: 49.26$ & $59: 13: 19.00$ & 20.065 & 0.034 & 1.237 & 0.064 & 0.545 & 0.072 \\
9 & $0: 18: 51.49$ & $59: 13: 29.70$ & 19.676 & 0.025 & 1.325 & 0.047 & 0.548 & 0.054 \\
10 & $0: 18: 52.90$ & $59: 16: 01.60$ & 19.710 & 0.027 & 1.015 & 0.046 & 0.560 & 0.056 \\
11 & $0: 18: 54.16$ & $59: 11: 14.50$ & 20.729 & 0.051 & 1.189 & 0.088 & 0.320 & 0.083 \\
12 & $0: 18: 56.95$ & $59: 23: 02.30$ & 19.733 & 0.027 & 0.934 & 0.048 & 0.632 & 0.061 \\
\hline
\end{tabular}

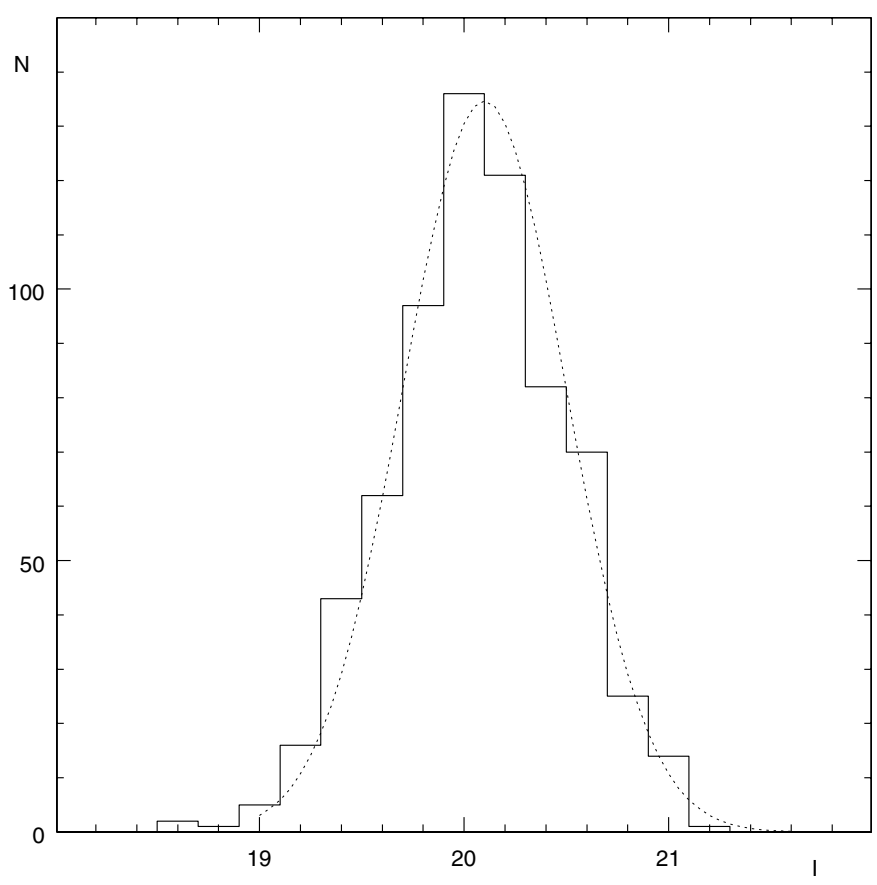

Fig. 6. Luminosity function of $\mathrm{C}$ stars. A Gaussian is eye fitted to the histogram.

a true modulus of $(m-M)_{0}=24.35 \pm 0.11$, corresponding to $741 \mathrm{kpc}$. This distance places IC 10 at about $240 \mathrm{kpc}$ from M31. The absolute magnitude of IC 10 is then $M_{v}=$ -15.6, adopting de Vaucouleurs \& Ables (1964) integrated apparent magnitude. Its luminosity is similar to NGC 3109 but brighter than NGC 6822 or IC 1613.

\subsection{Size of IC 10}

IC 10 has been often called the nearest starburst galaxy. It is even considered a "blue compact dwarf" by Richer et al. (2001). The recent planetary nebulae survey by Magrini et al. (2003) has however revealed that IC 10 must possess a much

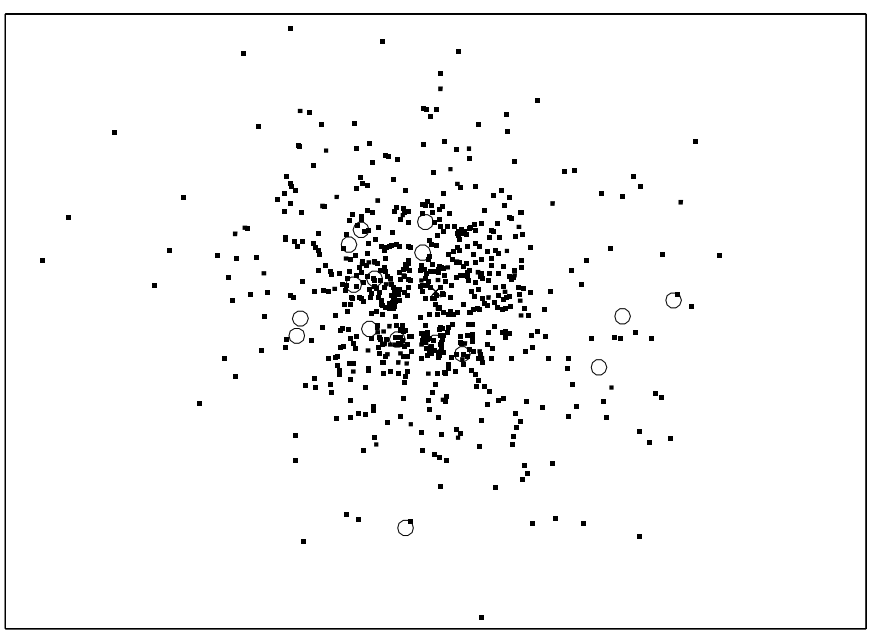

Fig. 7. The CFH12K field of IC 10 . The $676 \mathrm{C}$ stars are shown by solid dots while the known planetary nebulae are represented by circles. North is on top, East is on the left.

larger halo than the starburst core. Our C star survey confirms this finding and extends even further the distribution of the stellar halo. Figure 7 presents a map of the $\mathrm{C}$ stars and of the 16 known planetary nebulae. We see $C$ stars over most of the CFH12K 42' $\times 28^{\prime}$ field. The obvious central hole in the distribution corresponds to the densest core of IC 10 which can be seen in the $I$-band image published by Sakai et al. (1999). Note, however that their East-West orientation is reversed to ours. The horizontal gap and a less evident vertical one are due to $\sim 8^{\prime \prime}$ gaps between CCDs.

In order to determine the size of IC 10 we can use two tracers to study the surface density behaviour: 1) the bulk of the IC 10 stars, seen in the CMD of Fig. 4 or 2) C stars identified in Sect. 3.2. While the former sample consists in a much larger number of stars ( 20000 stars) the latter has the advantage of being free from foreground contamination. 


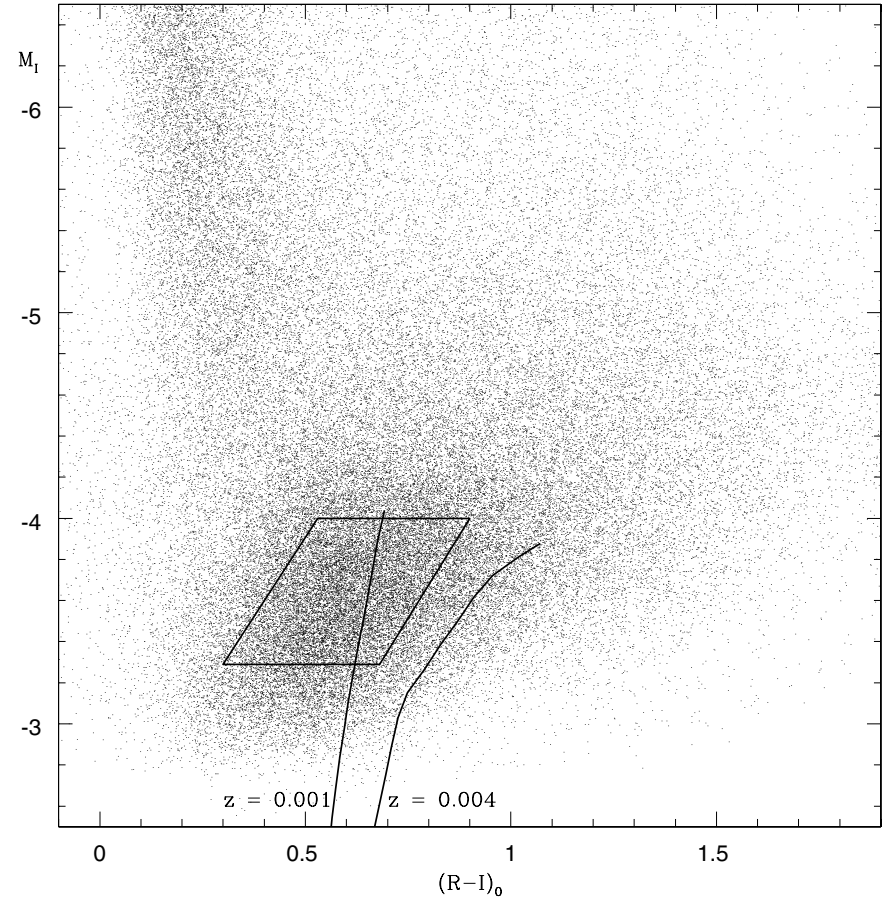

Fig. 8. A close-up of the CMD presented in Fig. 4 with $10 \mathrm{Gyr}$ isochrones with different abundances. The parallelogram defines the sample of red giants.

One of the major problem is to determine the center of the IC 10 because the galaxy is highly patchy near the center. For the following analysis we assume that: a) the center of the IC 10 corresponds to the coordinates given in NED; b) both the bulk of red stars and $\mathrm{C}$ stars have a spherical distribution around this center.

\subsubsection{Size from red giant stars}

Having determined the reddening and the distance of IC 10 we are now in position to establish the evolutionary status of the the bulk of its giants seen in our CMD. Since the oxygen abundance of IC 10, determined from its HII regions by Garnett (1990), is $\log (\mathrm{O} / \mathrm{H})+12=8.26$ we select Girardi et al. (2002) isochrones for $10 \mathrm{Gyr}$ and $Z=0.004$. The isochrone, as can be seen on a close-up of the CMD, displayed in Fig. 8, does not match the RGB location. A lower abundance, $z=0.001$, fits better the bulk of the observed RGB. Hunter (2001) has determined the mean colour of the TRGB to be $(V-I)_{0}=1.74$, this corresponds to $(R-I)_{0}=0.77$ using Battinelli et al. (2003) relation. Therefore, $z=0.001$ is obviously much more representative of the old population abundance. The parallelogram outlines the $\sim 15000$ stars we select as representative of the bulk of the old stellar population seen in our images.

The spatial distribution of these RGB stars is shown by isodensity contours displayed in Fig. 9. They clearly reveal how complex and irregular the structure of IC 10 is. In particular, all the northern half is highly asymmetric, in the inner as well as outer regions. Such irregularities cannot be ascribed to an extra dust absorption not properly accounted by our dereddening procedure (described in Sect. 3.1) since the average

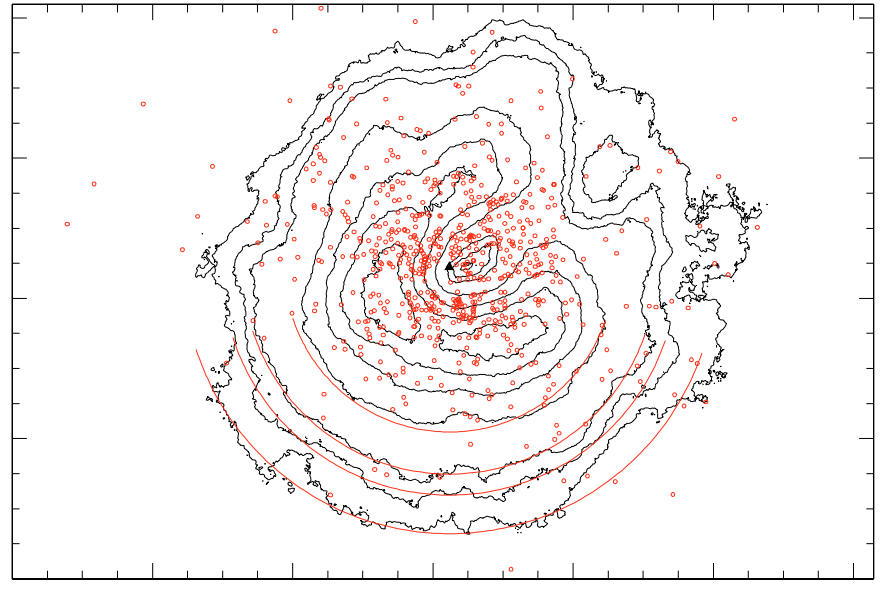

Fig. 9. Isodensity contours of red stars in IC 10 (see text) over the $42^{\prime} \times 28^{\prime} \mathrm{CFH} 12 \mathrm{~K}$ field of view. Contours are well represented by circular arcs in the Southern half of the galaxy.

$(R-I)_{0}$ for the $\mathrm{C}$ stars in the Northern and Southern halves of the galaxy are identical. Certainly, at least in the NW quarter of IC 10, the presence of a cospicuous population of young bright stars may prevent the detection of the fainter RGB component thus resulting into an artificial lower surface stellar density. On the other hand, the southern half appears much more regular and the contours, at least in the outer regions, are well matched by circular arcs centered on the adopted IC 10 center. These arcs demonstrate that the adopted center and the shape are well justified. We have to stress however that it is not possible to judge if a slight ellipticity is present.

The RGB stars in the box of Fig. 8 are used to derive the surface density in circular annulii, for the southern half of the galaxy. This profile is shown in Fig. 10. IC 10 seems to extend up to $\approx 16^{\prime}$ where a clear plateau, corresponding to the foreground, is reached. The average density of the last 8 points leads to a foreground density of $3.10 \pm 0.28 \mathrm{arcmin}^{-2}$. A radius of 16 arcmin, at the distance of IC 10 corresponds to a diameter of $7 \mathrm{kpc}$, making IC 10 one of the largest dwarf irregular of the Local Group.

From a least square fit of the foreground corrected logarithmic counts (see Fig. 11) we estimate a radial scale length of $2.59 \pm 0.06 \operatorname{arcmin}(560 \mathrm{pc})$. We remind again that such scale length refers only to the southern half of IC 10 and that the galaxy is much more irregular and possibly is less extended on the northern side (see Fig. 9).

\subsubsection{Size from C stars}

The mean $X$ and $Y$ coordinates of the $676 \mathrm{C}$ stars correspond to a point $35^{\prime \prime}$ off the presumed center, again justifying our adopted center. Since the foreground contamination is essentially nil for $\mathrm{C}$ stars we can derive the radial scale length directly from the observed counts. Similarly to the previous subsection, we limit our analysis to the southern half of the galaxy. The radial surface density profile is displayed in Fig. 11. The surface density, from $4^{\prime}$ to $15^{\prime}$ follows closely a power law. 


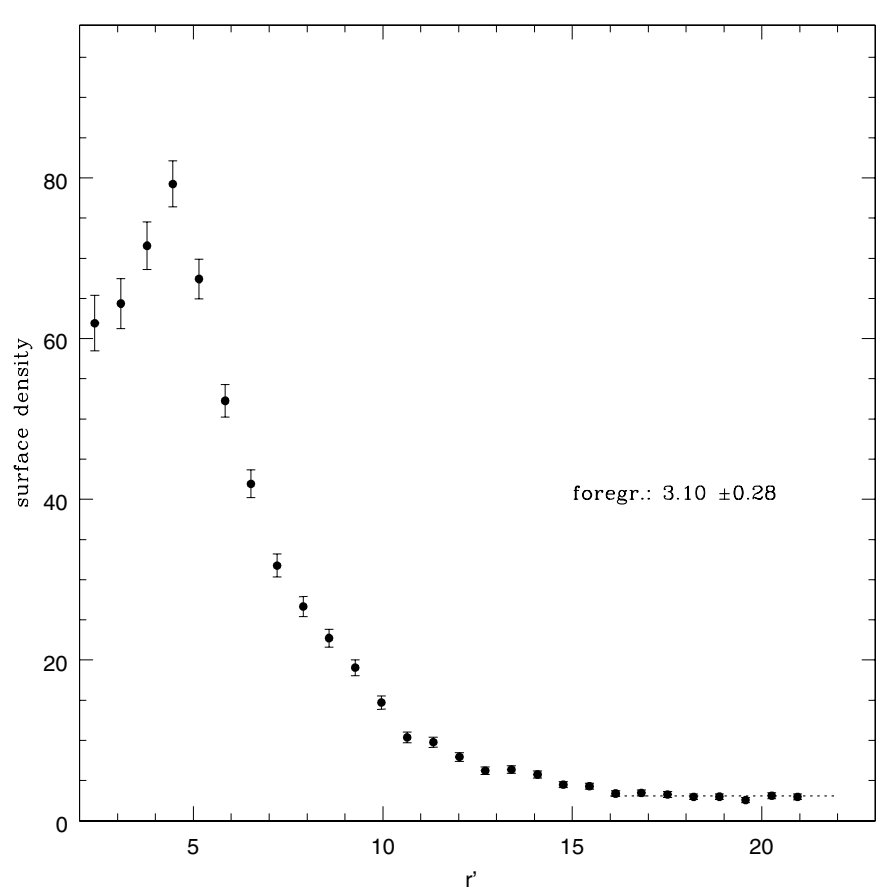

Fig. 10. Density profile (stars per $\operatorname{arcmin}^{2}$ ) from RGB star counts in the southern half of IC 10. The dotted line indicates the foreground density plateau.

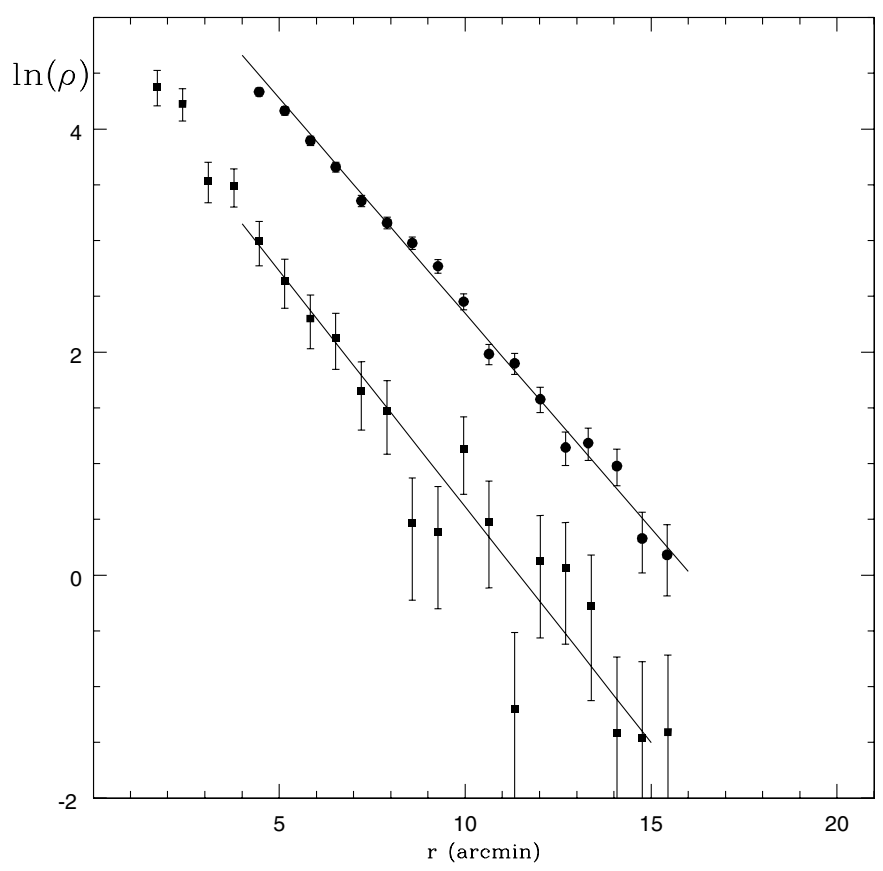

Fig. 11. Radial profile of the surface densities of old giants (filled circles) and C stars (squared dots), For an easy comparison 2 has been added to the logarithmic density of $\mathrm{C}$ stars. Surface density units are star $\operatorname{arcmin}^{-2}$. Lines are least square fits to the points in the radial range $4^{\prime}$ to $15^{\prime}$.

A least square solution through these points yields a radial scale length of $2.36 \pm 0.15^{\prime}$. This scale length, which is in fair agreement to what obtained in the previous subsection, implies that the old and the intermediate-age populations are - at least up to a radius of 15 arcmin - spatially distributed in the same way.

\subsection{The $C / M$ ratio}

To select AGB M stars among the stars in the M-box of the colour-colour diagram in Fig. 5, we follow the procedure adopted by Battinelli et al. (2003) which consists in rejecting stars with $M_{\text {bol }}$ fainter than the TRGB luminosity as well as those brighter than the brightest members of the galaxy. The bright cutoff for the selection of AGB M stars is $I_{0}=18.5$ which corresponds to the value used by Battinelli et al. (2003) for M31 scaled for the the different distance and absorption of IC 10. The whole CFH12K area contains 11204 such AGB stars. We evaluate the foreground contribution by assuming that two $5^{\prime}$ wide strips at both extremities of the field are essentially free of IC 10 stars. From these counts we estimate the total foreground contribution to be $6941 \pm 166$ stars resulting in $2993 \pm 166 M$ AGB stars belonging to IC 10 . The $C / M$ ratio is then $0.23 \pm 0.02$. A comprehensive discussion of the $C / M$ ratios as a metallicity indicator will be presented in the next paper of this series.

\section{Conclusions}

The use of the average $I$-luminosity of C stars as standard candles has provided one more estimate for the controversial distance to IC 10. The deduced value of $741 \mathrm{kpc}$, when compared with the values listed in Table 1, turns out to be exactly the average value of the 8 distance determinations published in the last decade. Local Group dwarf irregular galaxies are quite different in several respects. This investigation of IC 10 has, however, emphasizes its similarities with NGC 6822 whose $900 \mathrm{C}$ stars are distributed over at least a $42^{\prime} \times 28^{\prime}$ area (Letarte et al. 2002). Both galaxies have an extended HI envelope. The stellar halo of IC $10(\sim 7 \mathrm{kpc})$ is more extended that the one of NGC $6822(\sim 5 \mathrm{kpc})$. These galaxies are not the only dwarf irregulars with a substantial HI envelope, WLM (Huchtmeier et al. 1981) and NGC 3109 (de Blok \& Walter 2000) also present such gaseous component. However, these latter galaxies, contrary to IC 10 and NGC 6822, do not possess any significant stellar halo (Battinelli \& Demers 2004a; Demers et al. 2003). In all nearby galaxies that have been studied to deep enough magnitude limits, RR Lyrae stars have been found, i.e. all these galaxies seem to be embedded in old envelopes. Our $\mathrm{C}$ star survey has so far shown that the halos of dwarf irregular galaxies contain intermediate-age as well as old stars, generally with similar scale heights.

Acknowledgements. This research is funded in parts (S.D.) by the Natural Science and Engineering Council of Canada.

\section{References}

Alton, P. B., Trewhella, M., Davies, J. I., et al. 1998, A\&A, 335, 807 Alton, P. B., Lequeux, J., Bianchi, S., et al. 2001, A\&A, 366, 451 Battinelli, P., \& Demers, S. 2004a, A\&A, 416, 111 Battinelli, P., \& Demers, S. 2004b, A\&A, 417, 479 Battinelli, P., \& Demers, S. 2004c, A\&A, 418, 33

Battinelli, P., Demers, S., \& Letarte, B. 2003, AJ, 125, 1298

Borissova, J., Georgiev, L., Rasado, M., et al. 2000, A\&A, 363, 130

Bottinelli, L., Goughenheim, L., Paturel, G., \& de Vaucouleurs, G. 1984, A\&A, 56, 381 
Ciardullo, R., Jacoby, G. H., Ford, H. C., \& Neill, J. D. 1989, ApJ, 339,53

Cardelli, J. A., Clayton, J. C., \& Mathis, J. S. 1989, ApJ, 345, 245

Cox, A. N. 2000 in Allen's Astrophysical Quantities, 4th ed. (New York: Springer), 392

de Blok, W. J. G., \& Walter, F. 2000, ApJ, 537, L95

Demers, S., Dallaire, M., \& Battinelli, P. 2002, AJ, 123, 3428

Demers, S., Battinelli, P., \& Letarte, B. 2003, A\&A, 410, 795

Deul, E. R., \& Burton, W. B. 1992, in The Galactic Interstellar Medium, ed. W. B. Burton, B. G. Elmergreen, \& R. Genzel (Berlin: Springer-Verlag), Chap. 5

de Vaucouleurs, G. 1978, ApJ, 224, 710

de Vaucouleurs, G., \& Ables, H. 1964, PASP, 77, 272

Garnett, D. R. 1990, ApJ, 363, 142

Girardi, L., Bertelli, G., Bressan, A., et al. 2002, A\&A, 391, 195

Huchtmeier, W. K. 1979, A\&A, 75, 170

Huchtmeier, W. K., Seiradakis, J. H., \& Materne, J. 1981, A\&A, 102, 134

Hubble, E. 1926, The Realm of the Nebulae (New Haven: Yale U. Press), 126

Hunter, D. A. 2001, ApJ, 559, 225

Jacoby, G. H., \& Lesser, M. P. 1981, AJ, 86, 185

Karachentsev, I. D., \& Tikhonov, N. A. 1993, A\&AS, 100, 227

Lequeux, J., Peimbert, M., Rayo, J. F., Serrano, A., \& Torres-Peimbert, S. 1979, A\&A, 80, 155
Letarte, B., Demers, S., Battinelli, P., \& Kunkel, W. E. 2002, AJ, 123, 832

Massey, P., \& Armandroff, T. E. 1995, AJ, 109, 2470

Mayall, N. U. 1935, PASP, 47, 317

Méndez, R. A., \& Guzmán, R. 1998, A\&A, 333, 106

Richer, M. G., Bullejos, A., Borissova, J., et al. 2001, A\&A, 370, 34

Sadroski, T. J., Bennett, C., Boggess, N., et al. 1994, ApJ, 428, 638

Saha, A., Hoessel, J. G., Krist, J., \& Daneilson, G. E. 1995, AJ, 111, 197

Sakai, S., Madore, B. F., \& Freedman, W. L. 1999, ApJ, 511, 671

Sandage, A., \& Tammann, G. A. 1974, ApJ, 194, 559

Stetson, P. B. 1987, PASP, 99, 191

Stetson, P. B. 1994, PASP, 106, 250

Tikhonov, N. A. 1999, in The Stellar Content of Local Group Galaxies, ed. Patricia Whitelock, \& Russel Cannon (ASP), IAU Symp., 192

van den Bergh, S. 1999, ApJ, 517, L97

van den Bergh, S. 2000, The Galaxies of the Local Group, Cambridge Astrophys. Ser., 35

Wilson, C. D., Welch, D. L., Reid, I. N., Saha, A., \& Hoessel, J. 1996, AJ, 111, 1106

Xilouris, E. M., Alton, P. B., Davies, J. I., et al. 1998, A\&A, 331, 894 Xilouris, E. M., Byun, Y. I., Kylafis, N. D., Paleologou, E. V., \& Papamastorakis, J. 1999, A\&A, 344, 868

Yang, H., \& Skillman, E. D. 1993, AJ, 106, 1448 